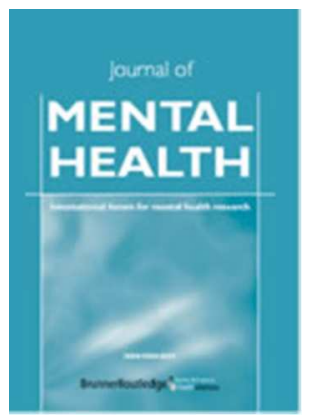

\title{
Delivery of cognitive-behaviour therapy for psychosis: A service user preference trial
}

\begin{tabular}{|r|l|}
\hline Journal: & Journal of Mental Health \\
\hline Manuscript ID & CJMH-2017-0009.R3 \\
\hline Manuscript Type: & Original Article \\
\hline Subject Area: & Mental Health \\
\hline Further Detail: & schizophrenia, CBT, psychosis, treatment preference, preference trial \\
\hline
\end{tabular}

\section{SCHOLARONE ${ }^{\text {m }}$}

Manuscripts 
Delivery preferences for CBT for psychosis

\title{
Delivery of cognitive-behaviour therapy for psychosis: A service user preference
}

\section{trial}

\begin{abstract}
Background: Clinical guidelines recommend cognitive behaviour therapy (CBT) for people with psychosis, however, implementation is poor and not everyone wishes to engage with therapy. Understanding service user (SU) preferences for receiving such treatments is a priority for services. Aims: To explore SU preferences and outcomes of different methods of delivering CBT for psychosis. Method: SUs experiencing psychosis could choose between treatment as usual (TAU); TAU plus telephone-delivered CBT with self-help, CBT recovery manual (TS); high support CBT (HS - TAU plus TS plus group sessions); or randomisation. Participants received their option of choice and were followed up on several outcomes over nine and 15 months.

Results: Of 89 people recruited, three chose to be randomised and 86 expressed a treatment preference ( 32 chose TAU, 34 chose TS, 23 chose HS). There were few differences between those that chose therapy compared to those that chose TAU. Those who had more positive impacts from their symptoms were significantly more likely to choose TAU.

Conclusions: Most people had strong preferences about treatment delivery and a substantial number did not wish to receive additional therapy. These findings have to be considered when planning and allocating resources for people with psychosis.

Declaration of interest: None

Keywords: schizophrenia, CBT, treatment preference, psychosis, preference trial
\end{abstract}


Delivery preferences for CBT for psychosis

Clinical guidelines recommend psychological therapies, such as cognitive-behaviour therapy (CBT), to support recovery for individuals with psychosis or schizophrenia (American Psychiatric Association, 2004; National Institute for Health and Care Excellence, 2014). However, implementation of CBT for psychosis (CBTp) is poor (Haddock et al., 2014; Prytys et al., 2011) and there are significant barriers to delivery within services, such as workforce capacity (Berry \& Haddock, 2008), service structure and service user (SU) preferences. The evidence base for CBTp has mainly been for face to face CBT (individual or group), although we know that CBT delivered by telephone is effective and acceptable as face-to-face delivery (Lovell et al., 2006). Despite this, preferences for delivery of CBTp are not well researched, although preferences about treatment have been explored for other mental health problems. This work has suggested that therapy may be more acceptable and credible than pharmacological interventions (Landreville et al., 2001; Lundervold \& Lewin, 1990; Rokke \& Scogin, 1995; Unützer et al., 2002), and that there are better outcomes for those receiving a treatment of choice (Swift \& Callahan, 2009).

Although little research has been done on preferences for CBTp, some studies suggest that people have strong opinions about therapy and how it is delivered (Sumner et al, 2014; Byrne et al, 2010; Byrne et al, 2014). In one study, which explored hypothetical preferences about delivery of CBTp, SUs were asked, if they were to be offered a treatment, how they would prefer therapy to be delivered with four possible options: CBTp by telephone, telephone CBTp plus group sessions, both including the support of a CBTp-focused guided self-help manual, 'no therapy' (TAU) or 'no preference' (randomly allocated to any treatment; Sumner et al., 2014). All were also asked whether they would be willing to take part in a trial. Of 81 participants, $97 \%$ were willing to be involved in such a trial, however, $57 \%$ were not be willing to be randomised and had preferences about how they would like to receive CBTp. Twenty two per cent chose TAU alone, $43 \%$ chose 
Delivery preferences for CBT for psychosis

telephone $\mathrm{CBT}$, and $27 \%$ preferred telephone therapy plus group sessions. Only one participant had no preference suggesting important considerations for service deliverers. For example, SUs may refuse treatment in case they do not receive their preferred option, or, they may drop out because they are unhappy with their allocation. In addition, although there were no sociodemographic or clinical differences between preference groups in the Sumner et al study, other studies have shown that preferences about treatment are associated with patient characteristics (Yung et al, McHugh et al, 2013). More information about this may help services to plan delivery of treatment based on specific characteristics.

To take account of treatment preferences, partially randomised Patient Preference Trial (PPT) designs are used (Brewin \& Bradley, 1989). People with treatment preferences are allowed their treatment without randomisation and those without a preference are randomised in the usual way. There are methodological problems with this design, e.g. any comparison that uses nonrandomised groups is potentially unreliable because of unknown confounds. However, there are ways of reducing the impact of this by performing a comparison of the randomised participants alone, collecting information on confounds and adjusting for these in the analysis and using randomisation status as a covariate in analyses (Torgerson \& Sibbald, 1998). Prospective investigations to ascertain expected numbers in each arm, and presenting information to participants about choices in a standardised way to minimise expectancy bias can help inform the design of such a trial. This methodology was chosen in the current study on the basis that the potential benefits outweighed the methodological problems. Benefits included the likelihood that we might recruit people who would otherwise refuse to take part in a traditional Randomised Controlled Trial (RCT), thus the sample would be more representative. Also, a PPT might improve the external validity of the trial i.e. be more commensurate with routine practice. We currently 
Delivery preferences for CBT for psychosis

have little information about treatment preferences in mental health and initiatives to bring parity for mental health care with physical health suggests that services should offer psychological therapy to everyone with psychosis (see National Institute for Health and Care Excellence - Quality Standard, 2015). Data on the likely uptake of therapy is important as to facilitate modelling of capacity and demand even though research data is not able to completely model 'real' service delivery. This information may help to overcome problems with implementation of psychological treatments for people with psychosis (Haddock et al., 2014). Hence, we aimed to examine SU characteristics in relation to treatment choice within the context of a real trial (in contrast to the hypothetical study of Sumner et al, 2014) and explore how this related to feasibility and acceptability of treatment, and explore outcomes following choice on recovery, symptoms and functioning. We anticipated that the characteristics of those choosing different options may vary which may allow us to deliver treatments matched to service user characteristics.

\section{Materials and Methods}

\section{Participants}

Participants were recruited from three National Health Service mental health trusts in the North West of England. Inclusion criteria were: ICD-10 (World Health Organisation, 1992) diagnosis of a schizophrenia spectrum disorder; 18-65 years; under care of a mental health team; use of a telephone, able to read English and provide informed consent, and stable symptoms for at least one month (defined as an absence of acute exacerbations of symptoms requiring hospitalisation or change in medication).

\section{Randomisation and masking}


Delivery preferences for CBT for psychosis

Following baseline assessment, participants took part in a semi-structured interview to ascertain preferences for treatment following presentation of a short description of each option. Participants were offered TAU alone (TAU), TAU plus telephone delivered CBT plus a supported self-help manual (TS), TAU plus TS plus recovery focused CBT group sessions (HS) or randomisation to any option. Options were presented in a counterbalanced order to remove order effects. Participants were asked about reasons for choice and the strength of choice (1-10, 10 being strongest). Participants were then allocated to their preferred option. Randomisation was conducted remotely by an independent clinical trials unit using random permuted blocks.

\section{Procedures}

Ethical approval for the study was provided by NRES Committee North West - Greater Manchester North (09/H1011/81; ISRCTN50487713). Participants were given $f 10$ for each assessment at baseline, post-therapy ( 9 months) and at 15 months. All assessments were carried out by research assistants blind to allocation.

\section{Intervention}

Further details of each treatment option:

1. Treatment as usual (TAU) consisted of care from a multi-disciplinary mental health team, prescriptions for antipsychotic medication, supportive information about medication and regular review.

2. Telephone support (TS) comprised weekly, telephone delivered CBTp with a supported selfhelp Recovery Guide delivered over 9 months. One initial and final face-to-face meeting were also offered. The Recovery Guide had two core chapters (Meaning of recovery from psychosis and goals, resources and understanding development of problems) and two 
Delivery preferences for CBT for psychosis

further chapters with optional sections (anxiety, paranoia, low self-esteem) or reaching wider recovery goals (employment, healthy living, social networks). The guide was developed by academics, clinicians and service user researchers. Participants could work on the guide independently, and within telephone sessions with a dedicated therapist who carried out a CBTp intervention. The intervention was intended to provide an equivalent amount of therapy as that which be carried out in usual face to face CBT for psychosis and was based on CBT psychosis interventions used in previous trials by the authors (Lewis et al, 2002; Barrowclough et al, 2010; Haddock et al, 2009) which informed UK, NICE guidelines for the delivery of CBT for people with psychosis (NICE, 2014). Telephone delivery was based on procedures developed in a CBT equivalence trial carried out by the first author (Lovell et al, 2006). All seven therapists met British Association for Behavioural and Cognitive Psychotherapies minimum training standards for CBT. Treatment fidelity was ensured through a detailed therapy manual, fortnightly group supervisions, individual therapist supervision and assessment of audio taped therapy sessions using a therapy fidelity scale (Hartley et al., 2014).

3. High Support (HS) consisted of TAU plus telephone delivered CBTp therapy as above plus 12 recovery focused CBTp group sessions delivered over six months, fortnightly, lasting two hours. Groups were run by a CBT therapist and a researcher with lived experience of psychosis. The sessions were CBT-based and matched chapters in the guide. Groups were aimed at encouraging participants to identify areas they would like to change and facilitated discussion around key CBT strategies. The treatment protocols closely matched that of traditional CBTp in groups (Barrowclough et al, 2006). Therapy adherence to the group intervention was ensured through using a detailed manual and supervision sessions as above. 
Delivery preferences for CBT for psychosis

In addition, some participants were offered peer support sessions delivered by service userconsultants. Up to six peer support sessions were offered, the first in person and the rest by telephone. However, due to resources, these sessions were only offered to the first 21 participants $(13=\mathrm{TS}, 8=\mathrm{HS})$.

\section{Outcomes}

Participants' choice and their ratings on strengths of choices (using Likert scales) were recorded during interview by the researcher. Reasons for choices were recorded verbatim. Data on take up of therapy sessions, time spent in therapy and therapeutic alliance were recorded. Demographic characteristics of participants and details of symptoms were recorded.

\section{Self-report mental health measures}

1. Questionnaire about the Process of Recovery (QPR; Neil et al., 2009): two sub-scales relating to interpersonal and intrapersonal recovery (22 items).

2. Subjective Experiences of Psychosis Scale (SEPS; Haddock et al., 2011): three subscales assessing the impact of psychotic symptoms, external factors and severity of psychotic symptoms (45 items).

\section{Secondary measures}

Structured interviews were used to measure symptom severity using the Positive and Negative Syndrome Scale (three subscales covering positive, negative and general symptoms, and a total score; PANSS; Kay et al., 1987) and Psychotic Symptom Rating Scales (an auditory hallucinations and delusions subscale score and a total score; PSYRATS; Haddock et al., 1999). Functioning was 
Delivery preferences for CBT for psychosis

measured by the total score of the informant rated Personal and Social Performance Scale (PSP; Morosini et al., 2000). Depression was assessed using the total score of informant rated Calgary Depression Scale (Addington et al., 1993) The self-report Beck Anxiety Inventory (BAI; Beck et al., 1988) and Self Esteem Rating Scale (Lecomte et al., 2006) total scores assessed anxiety and depression. All outcome measures have been widely used in psychosis samples and have good psychometric properties.

In addition, socio-demographic information (ethnicity, living arrangements, employment, education, marital status, religious beliefs) were assessed at baseline. Therapeutic alliance was measured by the self-report, client and therapist version of the Working Alliance Inventory (WAl; Horvath \& Greenberg, 1989) after the third telephone session. This has been widely used in psychosis samples and has good psychometric properties.

All assessors received training in the measures to a gold standard. The gold standard was developed in two previous trials of CBT for psychosis (Lewis et al, 2002 Barrowclough et al, 2010). Assessors had monthly supervision, where rating ambiguities were discussed and agreed. Inter-rater reliability was assessed quarterly using randomly selected cases. Average intraclass correlations from these analyses were .85 for the PANSS, .98 for the PSYRATS, .98 for the PSP and .92 for the CDS. All symptom measures were administered by blind assessors at baseline, nine months (end of treatment) and 15 months (follow-up).

\section{Statistical analysis}


Delivery preferences for CBT for psychosis

Reasons for treatment choices were analysed using content analysis (Hsieh \& Shannon, 2005) involving categorising participants' reasons into mutually exclusive themes. This was carried out by two researchers including one with lived experience of psychosis.

We performed analyses on participant demographic characteristics (age, gender, diagnosis, living arrangements, service type, employment), baseline PANSS, PSYRATS, SEPS, PSP and whether they had experienced previous psychological therapy in relation to treatment preferences. We used exploratory analysis to look at differences in therapy uptake between therapy groups, and also examined therapeutic alliance (TA), using therapist and participant reported WAI (reported in Mulligan et al., 2014).

The outcome analysis evaluated the effects of the interventions and assessed whether they were unaffected by the mechanism of treatment selection. As only 3 participants chose randomisation, this was not carried out. Outcome data were analysed using appropriate regression models to assess effects of treatment receipt, regardless of the method of treatment selection, using several baseline covariates (baseline value of the outcome measure being analysed, baseline PANSS Positive, PSP totals, sex, age, and education) to allow for possible confounding effects. Analyses were carried out using Stata version 11 (StataCorp, 2009). These outcomes are reported in full in Morrison et al (2016).

\section{Results}

\section{Sample}

244 participants were identified as eligible, and, of those, 206 were approached by their care coordinator to discuss participation (see Figure 1). 
Delivery preferences for CBT for psychosis

[Insert Figure 1 here]

Of these, 163 people agreed to be approached by the researcher, 25 did not respond to invitation to discuss participation, 43 refused to take part, leaving 95 people recruited. Of those, six withdrew from the study prior to therapy allocation. Demographic information is provided in Table 1.

[Insert Table 1 here]

\section{Preferences for delivery of psychological therapy}

Only three participants wished to be randomly assigned. A substantial proportion chose to receive TAU alone. The majority chose the TS option whilst a slightly smaller proportion chose the HS option (see Table 2). There were no significant differences in the strength of preferences between any of the three groups (see Table 3).

[Insert Tables 2 and 3 here]

\section{Reasons for choice}

All reasons for choice were analysed using content analysis and categorised independently by two raters (SH and LP) into mutually exclusive themes using the coding framework reported by Sumner and colleagues (2014) as a guide. The percentage agreement between raters was calculated representing the proportion of statements where total agreement was reached. The agreement for each of the categories was: willingness to participate $-84.4 \%$, unwillingness to participate $-69.2 \%$, 
Delivery preferences for CBT for psychosis

preference for TAU - 70.6\%, preference for TS - 91.2\%, preference for HS - 73.8\%, willingness to be randomised $-76.7 \%$, and unwillingness to be randomised $-84.4 \%$ (see Table 4 ).

\section{[Insert Table 4 here]}

In relation to choice of HS, reasons related to offering the opportunity to engage with others with similar mental health problems or for social contact. For others, HS was seen as an opportunity to get more therapy. For TS, reasons related to convenience of delivery e.g. being able to take advantage of therapy but not having to travel to clinics. Some reported that, while they wanted therapy, their problems were not severe enough to require additional group sessions. Others reported a dislike for groups, whilst still wishing to receive therapy. Those that chose TAU alone reported that therapy was unnecessary and expressed satisfaction with current services. Some cited reasons for choice directly related to mental health problems e.g. paranoia making it difficult to attend groups. Other cited reasons such as wanting to understand their illness and learning to relax as motivators for therapy. A small number reported that they wished to be involved in research for altruistic reasons but did not wish to take part in therapy.

\section{Preferences, demographics and symptoms \\ Differences between those who chose therapy $(L S+H S)$ and TAU}

We examined the differences in demographic characteristics between those who chose therapy versus TAU (i.e., age; gender; ICD-10 diagnosis; living arrangement; relationship status; type of previous and current therapy received (none vs. CBT); service type (early intervention vs. community care); employment status; and clinical measures (baseline QPR, BAI, BHS, SERS, SEPS, CDS, PANSS, and PSYRATS). There were no significant differences between participants' 
Delivery preferences for CBT for psychosis

demographic characteristics and their treatment preference. However, there were significant differences in BAI, SEPS negative scale, CDS, and PANSS negative scale scores. Those who chose therapy, rather than TAU, had more negative impact from psychosis symptoms (SEPS; $U=565.5, p$ $=.01$ ), were more anxious (BAI; $U=570, p=.01$ ), more depressed (CDS; $U=533, p=.004$ ), and experienced more negative symptoms of psychosis (PANSS; $U=624, p=.04$ ). There were no significant differences between those who choose TS and those who chose HS on demographic or clinical measures.

\section{Therapy uptake}

Descriptive statistics for therapy uptake are in Table 5. The TS group completed significantly more telephone sessions overall compared to the HS group $(F(1,55)=4.72, p=.034)$. There was a high level of variability in therapy attendance, with a range of 1-30 telephone support sessions attended and a range of $0-11$ group sessions attended.

Therapeutic alliance (TA) was comparable to previously reported in other CBTp trials. Consistent with previous studies (Barrowclough et al., 2010), therapist alliance scores were significantly lower than client-rated alliance (see Mulligan et al., 2014).

\section{[Insert Table 5 here]}

\section{Recovery and symptom outcomes following preferential allocation}

Linear regression models were fitted to estimate the treatment differences adjusted for age, sex, education and baseline scores for PANSS positive, PSP and corresponding baseline. There was no evidence of an overall treatment effect for the QPR total at nine months $(p=.58)$ and 15 months ( $p$ 
Delivery preferences for CBT for psychosis

$=.82$ ) or on any comparisons between the groups at either point. The same analyses were performed on SEPS. The only significant finding was for the SEPS negative impacts outcome at 15month follow-up, where there was an estimated difference in adjusted means comparing HS to TAU of 16.85 units ( $95 \% \mathrm{Cl} 1.36$ to $32.35, p=.03$ ) in favour of the control group. However, given multiple testing, this must be interpreted with caution. Similarly, there were no other secondary outcome differences between groups. (see Morrison et al., 2016 for a full description).

\section{Discussion}

Participants had strong preferences about treatment with most expressing a preference about treatment, rather than randomisation. Given the paucity of patient preference trials with people with psychosis, it is unclear whether this finding is generalizable, although other mental health trials have found higher rates of participants willing to be randomised (Bedi et al, 2000). This is important, given that little choice is usually offered for people with psychosis. Although the finding may be limited to CBTp, it suggests that preferences for other treatments may be an important topic for future research in this client group. The preferences varied slightly from those reported by Sumner and colleagues (2014) who found that $43 \%$ of participants were willing to be randomised, compared to just $3 \%$ in the present study, suggesting that asking people hypothetical questions about treatment preferences is not useful if we wish to know real figures for the purposes of a trial or service planning. We also found more people who opted for TAU rather than therapy, although overall treatment choices were similar across the three options.

The finding that a significant number of people did not choose to have therapy is important as the drive to improve access to CBTp assumes people all want it but cannot get it. There may have been other reasons for their refusal which we are not aware of, hence, fuller exploration of this may be 
Delivery preferences for CBT for psychosis

needed. However, it may suggest that more indirect working is warranted e.g. ensuring that individual's care is psychologically informed without providing one-to-one therapy. This has been shown to have some value in influencing outcomes (Berry \& Haddock, 2008; Berry et al., 2015). In addition, any participants who were in services were offered the opportunity to take part without consideration of whether they had been requesting therapy or not. This may well have influenced the findings. A future trial may examine choice and preference in a help seeking population which may provide different results.

Participants had varied reasons for their preferences and they were often strongly endorsed. Some of these related to personal preferences such as feeling satisfied with their current treatment or wanting someone to talk to. However, others expressed more external reasons, such as a desire to contribute to the research, rather than a desire to gain treatment. Clearly, the reasons for an individual's choice of treatment need to be carefully discussed with them. This may be an important consideration for rolling out CBTp interventions within routine services where SU treatment preferences are not always considered. This may result in poor take up of treatments which could, in part, account for the poor implementation of recommended psychological treatments for psychosis.

There were also no significant relationships between SU characteristics (e.g. diagnosis, age) and treatment preferences suggesting SUs' treatment choices cannot be easily predicted. It may therefore be important to offer treatment choices to SUs with psychosis on an individual basis, as the same modes of therapy may not be suitable for everyone.

\section{Outcome from treatment}


Delivery preferences for CBT for psychosis

There were no differences between groups in terms of treatment outcome. Group averages suggested that people within the study remained stable through to the 15 month follow-up, without significant improvement or deterioration, although this was variable with a wide range of outcomes across groups (see Morrison et al, 2016). It is possible that the sample recruited differed from those who would normally be recruited into psychological treatment trials, given that they were aware that they could choose their treatment option. What is unknown, is the effect of having treatment choice on outcomes. As such a small number chose to be randomised, it was not possible to fully explore outcomes against participants who were assigned to the treatment they may not have wanted. Hence, we do not know whether the outcomes would be different between those who received their choice compared to those who did not. This could only be overcome by having two samples i.e. one with randomisation only and one sample with choices.

\section{Limitations}

The findings are limited due to the PPT design and the low numbers choosing to be randomised, limiting the potential to make comparisons between groups. As a result, the findings are purely observational and, although conclusions can be made in relation to some aspects of the results, such as those relating to choice, it is not possible to make conclusions about specific outcomes from treatment. However, this method may be good for observational studies of clinical decision-making, especially if we consider the drive to improve delivery of psychological therapy i.e., we may be able to plan the provision of therapy just to those who want it. Hence, determining how many people want therapy and what is best suited to whom, is important. This could be enhanced by not just exploring uptake and engagement in therapy, but by exploring why people drop out or withdraw from therapy. In addition, we offered CBTp by telephone and group in this study, rather than traditional face to face therapy. Whilst, we know that telephone therapy has demonstrated 
Delivery preferences for CBT for psychosis

equivalence to face to face therapy in other patient groups, it's possible that this may not be the case in this sample. Further research could ensure this was explored together with other delivery options e.g. CBT delivered via internet or via app.

Finally, although we wished to deliver peer support as part of the treatment package, estimates of resources required to deliver this was below that which was actually required, resulting in this not being delivered to all participants as planned. As peer support is suggested in some guidelines (e.g. NICE), careful thought needs to be given to the resources required.

\section{Implications}

This study was the first of its kind with people experiencing psychosis and one of few conducted in mental health. It showed that SUs have strong preferences about treatment which are difficult to anticipate on participant characteristics alone. Although there were no significant differences between therapy groups in outcomes, the TS group was the most popular choice which may indicate that a similar format could be beneficial if refined and evaluated in a manner that permitted unconfounded analysis of treatment effects. Although telephone delivery of CBT has been little evaluated with this group, the findings showed it was acceptable and feasible with a similar therapeutic alliance to that observed in face-to-face CBT. Further research should explore several issues highlighted. For example, the effectiveness of telephone CBT in an RCT, feasibility and acceptability of peer support and its value in addition to $\mathrm{CBT}$, and further exploration of patient preferences and their impact on implementation.

\section{References}


Delivery preferences for CBT for psychosis

Addington, D., Addington, J., \& Maticka-Tyndale, E. (1993). Assessing depression in schizophrenia: the Calgary Depression Scale. British Journal of Psychiatry, Suppl. 22, 39-44.

American Psychiatric Association (2004). Practice guideline for the treatment of patients with schizophrenia ( ${ }^{\text {nd }}$ ed.). American Journal of Psychiatry, 161, 1-56.

Barrowclough, C., Haddock, G., Lobban, F., Jones, S., Siddle, R., Roberts, C. \& Gregg, L. (2006). Group Cognitive Behavioural Therapy for Schizophrenia. Randomised Controlled Trial. British Journal of Psychiatry, 189, 527-532.

Barrowclough, C., Meier, P., Beardmore, R., \& Emsley, R. (2010). Predicting therapeutic alliance in clients with psychosis and substance misuse. Journal of Nervous and Mental Disorders, 198, 373377.

Beck, A.T., Epstein, N., Brown, G., \& Steer, R.A. (1988). An inventory for measuring clinical anxiety: Psychometric properties. Journal of Consulting and Clinical Psychology, 56, 893-897.

Bedi, N., Lee, A., Harrison, G., Chilvers, C., Dewey, M., Fielding, K., Miller, P., Gretton, V., Williams, I., Churchill, R., Duggan, C. (2000) Assessing effectiveness of treatment of depression in primary care: Partially randomised preference trial. British Journal of Psychiatry, 177 (4) 312-318 
Delivery preferences for CBT for psychosis

Berry, K., \& Haddock, G. (2008). The implementation of the NICE guidelines for schizophrenia:

Barriers to the implementation of psychological interventions and recommendations for the future. Psychology and Psychotherapy: Theory, Research and Practice, 81, 419-436.

Berry, K., Haddock, G., Kellet, S., Roberts, C., Drake, R. and Barrowclough, C. (2015). Feasibility of a ward-based psychological intervention to improve staff and patient relationships in psychiatric rehabilitation settings. British Journal of Clinical Psychology. doi: 10.1111/bjc.12082.

Brewin, C.R., \& Bradley, C. (1989). Patient preferences and randomised clinical trials. British Medical Journal, 299, 313-315.

Byrne, R., Davies, L. and Morrison, A. (2010). Priorities and preferences for the outcomes of treatment of psychosis: A service user perspective. Psychosis, 2 (3), 210-217.

Byrne, R., \& Morrison, A. P. (2014). Service users' priorities and preferences for treatment of psychosis: a user-led Delphi study. Psychiatric services (Washington, D.C.), 65(9), 1167-9.

Haddock, G., Eisner, E., Boone, C., Davies, G., Barrowclough, C. (2014). An investigation of the implementation of NICE recommended CBT and FI interventions for people with schizophrenia. Journal of Mental Health, 23, 162-165.

Haddock, G., McCarron, J., Tarrier, N., \& Faragher, E.B. (1999). Scales to measure dimensions of hallucinations and delusions: The psychotic symptom rating scales (PSYRATS). Psychological Medicine, 29, 879-889. 
Delivery preferences for CBT for psychosis

Haddock. G., Barrowclough. C., Shaw. J., Dunn. G., Novaco. R. and Tarrier. N. (2009). Randomised controlled trial of cognitive behaviour therapy versus a social activity controlled treatment for people with psychosis and a history of violence. British Journal of Psychiatry, 194(2), 152-157.

Haddock, G., Wood, L., Watts, R., Dunn, G., Morrison, A.P., \& Price. J. (2011). The Subjective Experiences of Psychosis Scale (SEPS): Psychometric evaluation of a scale to assess outcome in psychosis. Schizophrenia Research, 133, 244-249.

Hartley, S., Scarratt, P., Bucci, S., Kelly, J., Mulligan, J., Neil, S.T. et al. (2014). Assessing therapist adherence to recovery-focused cognitive behavioural therapy for psychosis delivered by telephone with support from a self-help guide: Psychometric evaluations of a new fidelity scale. Behavioural and Cognitive Psychotherapy, 42, 435-451.

Horvath, A.O., \& Greenberg, L. S. (1989). Development and validation of the Working Alliance Inventory. Journal of Counselling Psychology, 36, 223-233.

Hsieh, H.F., \& Shannon, S.E. (2005). Three approaches to qualitative content analysis. Qualitative Health Research, 15, 1277-1288.

Jung, H.P., Baerveldt, C., Olesen, F., Grol, R., Wensing, M. (2003). Patient characteristics as predictors of primary health care preferences: a systematic literature analysis. Health Expectations, 6(2), 160-181. 
Delivery preferences for CBT for psychosis

Kay, S.R., Fiszbein, A., \& Opler, L.A. (1987). The Positive and Negative Syndrome Scale (PANSS) for Schizophrenia. Schizophrenia Bulletin, 13, 261-276.

Landreville, P., Landry, J., Baillargeon, L., Guerette, \& Matteau, E. (2001). Older adults' acceptance of psychological and pharmacological treatments for depression. Journal of Gerontology, 56B, 285291.

Lecomte, T., Corbière, M., \& Laisné, F. (2006). Investigating self-esteem in individuals with schizophrenia: Relevance of the Self-Esteem Rating Scale-Short Form. Psychiatric Research, 143, 99108.

Lewis, S., Tarrier, N., Haddock, G., Bentall, R.P., Kinderman, P., Kingdon, D. Siddle, R., Drake, R., Everitt, K., Leadley, K., Benn, A., Grazebrook, K., Haley, C., Akhtar, S., Davies, L., Palmer, S., Faragher, B. and Dunn, G. (2002). Randomised controlled trial of cognitive behaviour therapy in early schizophrenia: acute phase outcomes. British Journal of Psychiatry, 181 (sppl. 43), s91-s97.

Lovell, K., Cox, D., Haddock, G., Jones, C., Raines, D., Garvey, R., Roberts, C., \& Hadley, S. (2006).

Telephone administered cognitive behaviour therapy for treatment of obsessive compulsive disorder: randomised controlled non-inferiority trial. British Medical Journal, 333, 883-886.

Lundervold, D., \& Lewin, L. M. (1990). Older adults' acceptability of pharmacotherapy and behavior therapy for depression. Journal of Applied Gerontology, 9, 211-215. 
Delivery preferences for CBT for psychosis

McHugh, R.K., Whitton, S.W., Peckham, A.D., Welge, J.A., Otto, M.W. (2013). Patient Preference for Psychological vs. Pharmacological Treatment of Psychiatric Disorders: A Meta-Analytic Review Journal of Clinical Psychiatry, 74 (6), 595-602.

Morosini, P.L., Magliano, L., Brambilla, L., Ugolini, S., \& Pioli, R. (2000). Development, reliability and acceptability of a new version of the DSM-IV Social and Occupational Functioning Assessment Scale (SOFAS) to assess routine social functioning. Acta Psychiatrica Scandinavica, 101, 323-329.

\author{
Morrison, A.P., Law, H., Barrowclough, C., Bentall, R.B., Haddock, G., Jones, S.H., Kilbride, M., Pitt, E., \\ Shryane, N., Tarrier, N., Welford M., \& Dunn., G. (2016). Psychological approaches to understanding \\ and promoting recovery in psychosis and bipolar disorder: a mixed-methods approach. Programme \\ Grants for Applied Research, 4, 1-272.
}

\begin{abstract}
Mulligan, J., Haddock, G., Hartley, S., Davies, J., Sharp, T., Kelly, J., et al. (2014). An exploration of the therapeutic alliance within a telephone-based cognitive behaviour therapy for individuals with experience of psychosis. Psychology and Psychotherapy, 87, 393-410.
\end{abstract}

Neil, S.T., Kilbride, M., Pitt, L., Nothard, S., Welford, M., Sellwood, W. et al. (2009). The questionnaire about the process of recovery (QPR): A measurement tool developed in collaboration with service users. Psychosis, 1, 145-155.

\begin{abstract}
National Institute for Health and Care Excellence - Clinical Guideline (2014). Psychosis and schizophrenia in adults: treatment and management, [CG178]. London: National Institute for Healthcare and Excellence.
\end{abstract}


Delivery preferences for CBT for psychosis

National Institute for Health and Care Excellence - Quality Standard. (2015). Psychosis and schizophrenia in adults. NICE quality standard, [QS80]. Quality statement 1: Referral to early intervention in psychosis services. London: National Institute for Healthcare and Excellence.

Prytys, M., Garety, P.A., Jolley , S., Onwumere, J., \& Craig, T. (2011). Implementing the NICE guideline for schizophrenia recommendations for psychological therapies: A qualitative analysis of the attitudes of CMHT staff. Clinical Psychology and Psychotherapy, 18, 48-59.

Rokke, P. D., \& Scogin, F. (1995). Depression treatment preferences in younger and older adults. Journal of Clinical Geropsychology, 1, 243-257.

StataCorp (2009). Stata Statistical Software: Release 11. College Station, TX: StataCorp LP.

Sumner, K., Haddock, G., Hartley, S., McCusker, M., Pitt, L., Woodward, S. et al. (2014). Preferences for psychological therapy in psychosis: Trial participation, mode of treatment and willingness to be randomised. Journal of Mental Health, 23, 67-71.

Swift, J.K. \& Callahan, J.L. (2009) The impact of client treatment preference on outcome: A metaanalysis. Journal of Clinical Psychology, 65(4), 368-381.

Torgerson, D., \& Sibbald, B. (1998). Understanding controlled trials: what is a patient preference trial? British Medical Journal, 316, 360. 
Delivery preferences for CBT for psychosis

Unützer, J., Katon, W., Callahan, C. M., Williams, J. W., Jr., Hunkeler, E., Harpole, L., Langston, C. (2002). Collaborative care management of late-life depression in the primary care setting. Journal of the American Medical Association, 288, 2836-2845.

World Health Organization. (1992). The ICD-10 classification of mental and behavioural disorders: Clinical descriptions and diagnostic guidelines. Geneva: World Health Organization.

\section{Acknowledgements}

Acknowledgement to the Service User Consultants, (named alphabetically) and individual members of the Service User Reference Group, Yvonne Awenat, Rory Byrne, Ellen Hodson, Sam Omar, Liz Pitt, Jason Price, Tim Rawcliffe and Yvonne Thomas, for their work on this study. We also acknowledge the Mental Health Research Network, the NHS trusts where the study took place (Manchester Mental Health and Social Care Trust, Greater Manchester West NHS Trust, Five Boroughs NHS Trust) and the significant contribution made in the early stages of the project by Martina Kilbride. Ethical approval for the study was provided by NRES Committee North West - Greater Manchester North, reference 09/H1011/81. Trial Identifier: ISRCTN50487713. We also wish to acknowledge the role of the National Institute for Health Research (NIHR) for funding this study. This report/article presents independent research commissioned by the National Institute for Health Research (NIHR) under its Programme Grants for Applied Research scheme (RP-PG-0606-1086). The views expressed in this publication are those of the authors and not necessarily those of the NHS, the NIHR or the Department of Health.

\section{Disclaimer}


Delivery preferences for CBT for psychosis

The authors wish to recognise that the terms and language used in this article such as service user/client/psychosis are not universally endorsed by all. Where differences of opinion arose in preparation of this work, the authors decided to use the term that was agreed by the majority, while also respecting the views of others. 
Delivery preferences for CBT for psychosis

Figure 1: CONSORT diagram to show participant flow throughout the trial. TAU - treatment as ususal; TS - Telephone Therapy; HS - High Support therapy.

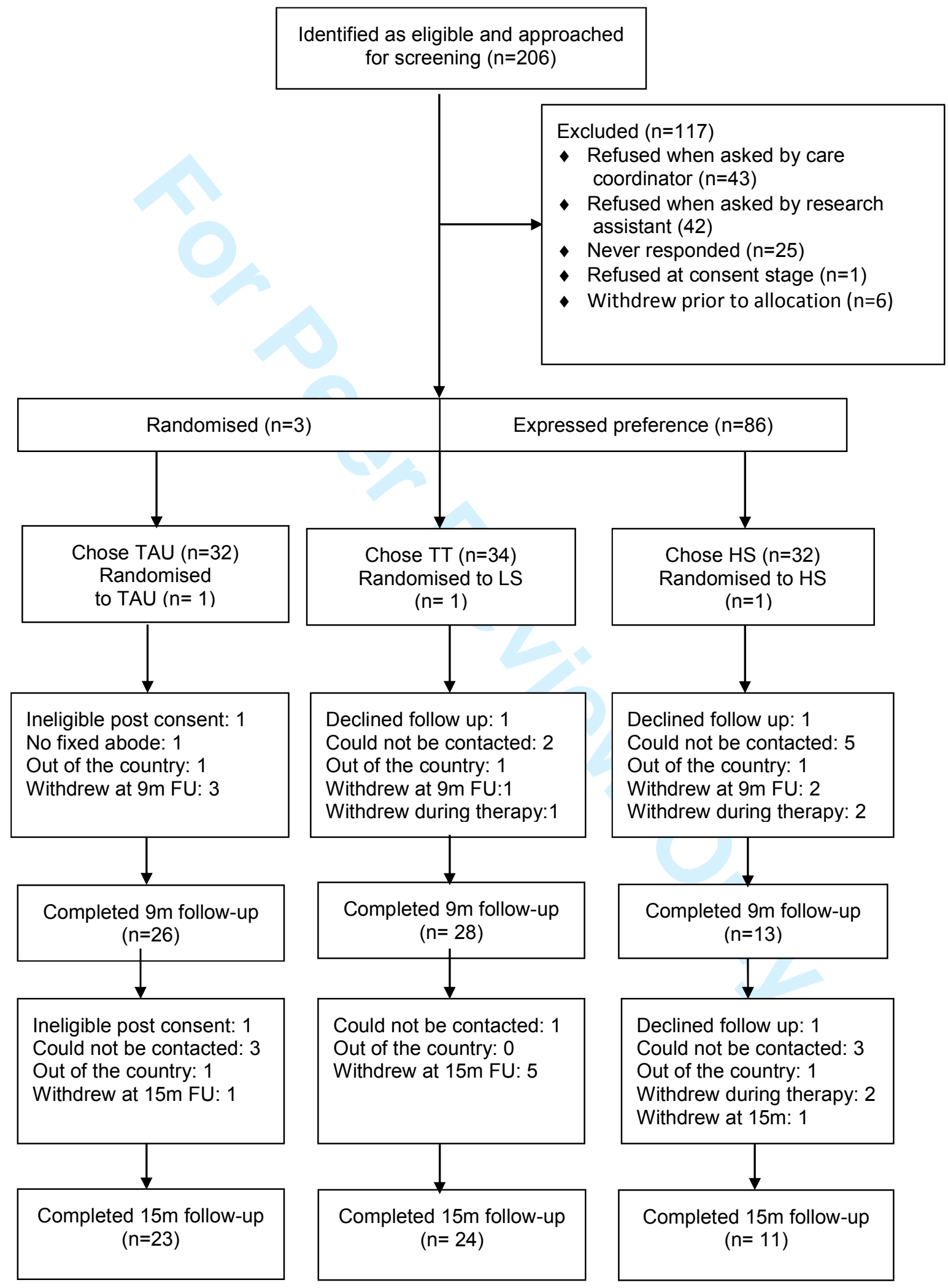


Delivery preferences for CBT for psychosis

Table 1: Sample demographics

\begin{tabular}{|c|c|c|c|c|c|c|c|c|c|}
\hline & & \multicolumn{2}{|c|}{ Total } & \multicolumn{2}{|c|}{ TAU } & \multicolumn{2}{|c|}{ TS } & \multicolumn{2}{|c|}{ HS } \\
\hline \multicolumn{2}{|c|}{$\begin{array}{l}\text { Participant characteristics at the } \\
\text { time of consent }\end{array}$} & $\mathrm{N}$ & $\%$ & $\mathrm{~N}$ & $\%$ & $\mathrm{~N}$ & $\%$ & $N$ & $\%$ \\
\hline Age & Mean (SD) & \multicolumn{2}{|c|}{$36(10.9)$} & \multicolumn{2}{|c|}{$35(11.2)$} & \multicolumn{2}{|c|}{$38(9.7)$} & \multicolumn{2}{|c|}{$33.3(10.6)$} \\
\hline \multirow{3}{*}{ Gender } & Male & 60 & 63 & 25 & 28.1 & 20 & 22.5 & 14 & 15.7 \\
\hline & Female & 35 & 37 & 8 & 9 & 13 & 14.6 & 9 & 10.1 \\
\hline & White & 76 & 80 & 26 & 29.2 & 26 & 29.2 & 21 & 23.6 \\
\hline \multirow[t]{5}{*}{ Ethnicity } & $\begin{array}{l}\text { Black/ Minority } \\
\text { Ethnic Group }\end{array}$ & 13 & 14 & 7 & 7.9 & 4 & 4.5 & 3 & 3.4 \\
\hline & Mixed race & 4 & 4 & 0 & 0 & 3 & 3 & 1 & 1 \\
\hline & Not reported & 2 & 2 & - & - & - & - & - & - \\
\hline & Employed/studyin & 24 & 73 & 9 & 10 & 6 & 7 & 6 & 7 \\
\hline & g & & & & & & & & \\
\hline \multirow{3}{*}{ Occupation } & $\begin{array}{l}\text { Not } \\
\text { working/studying }\end{array}$ & 69 & 25 & 24 & 27 & 27 & 30 & 17 & 19 \\
\hline & Not reported & 2 & 2 & - & - & - & - & - & - \\
\hline & Post-16 education & 51 & 44 & 19 & 21.3 & 18 & 20.2 & 11 & 12.4 \\
\hline \multirow[t]{3}{*}{ Education } & $\begin{array}{l}\text { No post-16 } \\
\text { education }\end{array}$ & 42 & 54 & 14 & 15 & 15 & 17 & 12 & 13 \\
\hline & Not reported & 2 & 2 & - & - & - & - & - & - \\
\hline & Alone & 60 & 63 & 21 & 2 & 18 & 20.2 & 19 & 21.3 \\
\hline \multirow{3}{*}{$\begin{array}{l}\text { Living } \\
\text { Arrangements }\end{array}$} & $\begin{array}{l}\text { With family } \\
\text { members/ partner }\end{array}$ & 31 & 33 & 12 & 13.5 & 14 & 15.7 & 4 & 4.5 \\
\hline & $\begin{array}{l}\text { Supported } \\
\text { accommodation }\end{array}$ & 2 & 2 & 0 & 0 & 1 & 1.1 & 0 & 0 \\
\hline & Not reported & 2 & 2 & - & - & - & - & - & - \\
\hline \multirow[t]{3}{*}{ Marital status } & $\begin{array}{l}\text { Married/civil } \\
\text { partnership/ } \\
\text { cohabiting }\end{array}$ & 10 & 11 & 4 & 4.5 & 4 & 4.5 & 2 & 2.2 \\
\hline & Single & 83 & 87 & 29 & 32.6 & 29 & 32.6 & 21 & 23.6 \\
\hline & Not reported & 2 & 2 & & & & & & \\
\hline \multirow{6}{*}{ Diagnosis } & Schizophrenia & 39 & 41 & 15 & 17 & 14 & 15.9 & 7 & 8 \\
\hline & $\begin{array}{l}\text { Schizoaffective } \\
\text { disorder }\end{array}$ & 12 & 13 & 2 & 2.3 & 5 & 5.7 & 2 & 2.3 \\
\hline & Psychosis NOS & 35 & 36 & 13 & 14.8 & 13 & 14.8 & 10 & 11.4 \\
\hline & Delusional disorder & 3 & 3 & 0 & 0 & 2 & 2.3 & 1 & 1.1 \\
\hline & $\begin{array}{l}\text { Paranoid } \\
\text { schizophrenia }\end{array}$ & 6 & 6 & 2 & 2.3 & 1 & 1.1 & 3 & 3.4 \\
\hline & Bipolar disorder & 1 & 1 & 0 & 0 & 0 & 0 & 0 & 0 \\
\hline
\end{tabular}


Delivery preferences for CBT for psychosis

1

2

3

4

5

6

7

8

9

10

11

12

13

14

15

16

17

18

19

20

21

22

23

24

25

26

27

28

29

30

31

32

33

34

35

36

37

38

39

40

41

42

43

44

45

46

47

48

49

50

51

52

53

54

55

56

57

58

59

60
Table 2: Therapy preferences and allocation

\begin{tabular}{cc}
\hline Allocations & Frequency (\%) \\
\hline Not allocated & $6(6)$ \\
TAU & $31(33)$ \\
Low support & $33(35)$ \\
High support & $22(23)$ \\
Randomised & $3(3)$
\end{tabular}


Delivery preferences for CBT for psychosis

Table 3: Strength of Preferences

\begin{tabular}{ccccccc}
\hline & \multicolumn{3}{c}{ Strength of } & \multicolumn{3}{c}{$\begin{array}{c}\text { Strength of } \\
\text { therapy preference }\end{array}$} \\
\hline & Mean & SD & Range & Mean & SD & Range \\
TAU & 8.58 & 1.38 & $5-10$ & n/a & n/a & n/a \\
TS & 8.3 & 1.31 & $5-10$ & 8.19 & 2.02 & $2-10$ \\
HS & 8.34 & 1.19 & $5-10$ & 8.24 & 1.09 & $5-10$ \\
\hline
\end{tabular}


Delivery preferences for CBT for psychosis

Table 4: Themes arising from analysis of reasons for treatment preference

Major themes (frequency of statements)

Examples

TAU (35)

1. Dislike of/lack of desire for aspect of therapy/ therapy in general (16)

'I don't want telephone therapy and wouldn't like a group'

'I don't like it on the phone, would prefer face to face'

2. Therapy viewed as unnecessary at present (8)

'I feel I'm doing so well, feel I don't need any other support at the moment'

'Feel I'm doing ok at the moment, don't want to jinx it'

'What I'm getting at the moment is good, in the past I

3.

Satisfaction with current support (10) didn't want help, now I receive a lot of help and this is working'

'I'm getting enough support at the moment- helping with practical support like finding work. Want to get out of psychiatric services'

4. Assist the research (1) 'Because I don't mind doing the questionnaires'

LS (39)

1. Dislike of/lack of desire 'Sounds like the group might be a lot of travelling and too for group support (23) much time' 'I'm very paranoid- don't like meeting new people so I wouldn't like the group'

2. Desire to receive 'I don't usually go out of the house so phone support might therapy/ therapy viewed be good' as helpful (13)

'The CBT calls- I can talk through problems' useful'

3. Desire to receive self- 'Get to do the Recovery Guide because I think it might be help manual (1) helpful'

4. Group support 'I would have chosen high support but I'm more well nowunnecessary (2) I'm past that stage, I don't need it.'

'...the high support would be a bit too much time for me' 
Delivery preferences for CBT for psychosis
Major themes (frequency of statements)
Examples

HS (74)

1. Social aspect/ normalising/ sharing experiences (11)

2. Benefits to self/highest level of support is most beneficial (8)

3 To gain knowledge/ information/ understanding (1)

4. For an experience/activity/ out of interest (4)

5. Desire for group support (2) 'I want to try out the group sessions' 'It's got the most activities to be involved in' they have and share problems' the same things'

'More stuff to help me' how to relax- want to get well' group sessions' psychological support'
It's a chance to meet people and discuss what support

'It would be good to meet other people who have similar experiences-I don't know anyone who has gone through

'Want to try and stabilise me- deal with the pressure of

'I want to understand it more- what triggers my illness,

'High support will get me out doing something within

'Don't usually get involved but like the idea of it an

31

32 
4

Delivery preferences for CBT for psychosis

Table 5: Therapy uptake

\begin{tabular}{lcc}
\hline & \multicolumn{2}{c}{ Therapy Group } \\
\cline { 2 - 3 } & Telephone & High Support \\
& mean (SD) & mean (SD) \\
\hline Number of telephone sessions attended & $15.82(10.77)$ & $9.78(9.54)$ \\
Number of group sessions attended & n/a & $2.96(3.60)$ \\
Total number of all sessions attended* & $13.39(10.64)$ & $12.68(12.92)$ \\
Total number of telephone session DNAs & $4.35(4.96)$ & $4.48(4.88)$ \\
Average telephone session length (minutes) & $40.56(9.23)$ & $39.55(1.32)$ \\
\hline * Group + telephone sessions. & &
\end{tabular}

* Group + telephone sessions. 